\title{
Conserved microstructure of the Brassica B Genome of Brassica nigra in relation to homologous regions of Arabidopsis thaliana, $B$. rapa and B. oleracea
}

Zahra-Katy Navabi ${ }^{1}$, Terry Huebert ${ }^{1}$, Andrew G Sharpe ${ }^{2}$, Carmel M O'Neill ${ }^{3}$, lan Bancroft ${ }^{3}$ and Isobel AP Parkin ${ }^{1 *}$

\begin{abstract}
Background: The Brassica B genome is known to carry several important traits, yet there has been limited analyses of its underlying genome structure, especially in comparison to the closely related $\mathrm{A}$ and $\mathrm{C}$ genomes. A bacterial artificial chromosome (BAC) library of Brassica nigra was developed and screened with 17 genes from a $222 \mathrm{~kb}$ region of $A$. thaliana that had been well characterised in both the Brassica A and C genomes.

Results: Fingerprinting of 483 apparently non-redundant clones defined physical contigs for the corresponding regions in B. nigra. The target region is duplicated in A. thaliana and six homologous contigs were found in B. nigra resulting from the whole genome triplication event shared by the Brassiceae tribe. BACs representative of each region were sequenced to elucidate the level of microscale rearrangements across the Brassica species divide.

Conclusions: Although the B genome species separated from the A/C lineage some 6 Mya, comparisons between the three paleopolyploid Brassica genomes revealed extensive conservation of gene content and sequence identity. The level of fractionation or gene loss varied across genomes and genomic regions; however, the greatest loss of genes was observed to be common to all three genomes. One large-scale chromosomal rearrangement differentiated the $B$ genome suggesting such events could contribute to the lack of recombination observed between $B$ genome species and those of the closely related $A / C$ lineage.
\end{abstract}

Keywords: Brassiceae, Brassica nigra, Sequence analyses, Speciation, Genome organization, Collinearity, Divergence time, Inversion

\section{Background}

Black mustard (Brassica nigra (L.) Koch, $2 n=16$, BB genome) represents the diploid Brassica B genome. It is an outcrossing oilseed species [1] which can also be used as a condiment [2]. However, it is a relatively minor crop compared with the closely related mustard species Brassica juncea (L.) Czern (AABB genome) and Brassica carinata Braun (BBCC genome), which are allotetraploid species that share the $B$ genome [1]. Most studies within the Brassiceae have focused on the widely cultivated Brassica species, Brassica rapa L. (AA genome), Brassica

\footnotetext{
* Correspondence: isobel.parkin@agr.gc.ca

${ }^{1}$ Agriculture and Agri-Food Canada, 107 Science Place, Saskatoon, SK S7N $0 \times 2$, Canada

Full list of author information is available at the end of the article
}

oleracea L. (CC genome), and Brassica napus L. (AACC genome) [3]. However, the diploid Brassica B genome is considered to be an important source of useful genes in Brassica breeding, including drought tolerance, disease resistance, and oil seed quality [4-9]. In order to exploit the variation found within the $B$ genome for the breeding of other Brassica oilseeds, an understanding of the relationship between the three Brassica genomes is required.

Although there has been extensive evidence of pairing and recombination between the $\mathrm{A}$ and $\mathrm{C}$ Brassica genomes [10-12] the $B$ genome appears unusual since no or limited recombination between the $\mathrm{B}$ genome and its related $\mathrm{A}$ and $\mathrm{C}$ homologues has been observed [13-17]. The reason for this difference can be hypothesized as

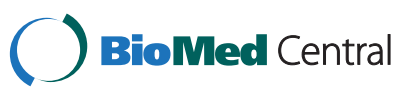


either the result of genetic factors regulating homologous pairing in the $\mathrm{B}$ genome similar to those suggested for the A and $C$ genomes [18-21] or significant structural divergence of the $\mathrm{B}$ genome relative to the $\mathrm{A}$ and $\mathrm{C}$ genomes. Although it has been suggested through limited sequence and comparative mapping data that the $\mathrm{B}$ genome may have diverged to such an extent that the lack of recombination can be explained [22], questions still arise as to accuracy of this assertion. Uncovering the level of homology at the micro level between the three Brassica diploid genomes should provide insights into the true relationship between the genomes.

The cultivated Brassica species are closely related to the fully sequenced model plant Arabidopsis thaliana, [23] since the Brassica and Arabidopsis lineages diverged between 14.5 and 20 million years ago (Mya) [24]. It is believed that $B$. nigra evolved from the same polyploidy events, and resultant paleohexaploid, which took place around 7.9 to 14.6 Mya within the Brassica lineage. Although there are good estimates placing the separation of the B. rapa (A genome) and B. oleracea (C genome) at 3.7 Mya [25], there is little data to age the separation of the $\mathrm{B}$ genome from the $\mathrm{A} / \mathrm{C}$ lineage [26]. Comparative mapping has identified blocks of conserved gene content and order between the A. thaliana genome and the Brassica A and C genomes of B. napus; where 21 ancestral segments of the $A$. thaliana genome could be replicated and rearranged to represent the Brassica genomes [27]. This work and further mapping in related species was used to propose an ancestral karyotype, reconstructed from 24 conserved blocks, A-X, for the entire Brassicaceae family [28]. Physical mapping of the regions homologous to a $222 \mathrm{~kb}$ region of $A$. thaliana chromosome 4 in $B$. oleracea [29], B. rapa [30] and B. napus [31] showed highly conserved collinearity of the orthologous regions in the three genomes and corroborated the triplicated nature of the diploid Brassica genomes. Previous sequence-level studies in B. oleracea [32], B. rapa [33] and $B$. napus [34] have clarified aspects of genome evolution and organization in Brassica by exploiting the close relationship with the genome of the model plant A. thaliana.

Physical mapping can provide an accurate representation of a genome when sequence is not available. A number of large insert libraries, the foundation for most physical maps, have been developed for the Brassica A and $\mathrm{C}$ genome species and have been exploited to facilitate map-based gene cloning for traits of interest and to provide insights into the evolutionary mechanisms that have formed these complex genomes [29-32,35-39]. In order to provide insights into the relatively uncharted Brassica B genome we have developed a large insert library for $B$. nigra and have used this tool to describe the physical organisation of six regions of the $B$ genome of
B. nigra homologous to the same $222 \mathrm{~kb}$ region of $A$. thaliana, which was exploited previously to uncover aspects of genome organisation and evolution in the Brassica A/C genomes [29,30,32,34]. We compared these orthologous regions of the $\mathrm{B}$ genome with the equivalent regions of $A$. thaliana, and the Brassica A and $\mathrm{C}$ genomes at the sequence level and report highly conserved gene content and order across the three genomes. An earlier divergence time separated the $\mathrm{B}$ genome from the $\mathrm{A}$ and $\mathrm{C}$ Brassica lineage by some 3 million years, which is barely reflected in the level of observed genome fractionation.

\section{Results}

\section{BGH BAC library characteristics}

In order to facilitate comparative mapping among the Brassica species a BAC library was constructed from a doubled haploid (DH) B. nigra line (No100). The $B$. nigra library $(\mathrm{BGH})$ was generated from HindIII digested genomic DNA and, includes 85,248 clones arrayed in 384 well plates. One clone was randomly selected from each of 100 plates. Restriction digestion of BAC DNA and resolution with pulse field gel electrophoresis allowed the average insert size to be estimated at $130 \mathrm{~kb}$. There were no empty clones identified among the 100 selected. In order to accurately assess the number of empty clones, 20 plates (7,680 clones) were gridded in replicate onto medium containing IPTG and X-gal, which resolved 141 (1.8\%) empty clones. The library should provide an approximate $17.5 \mathrm{X}$ coverage of the $\mathrm{B}$ genome, which is estimated to be $632 \mathrm{Mb}$ [40].

\section{Identifying the B genome BACs homologous to the $A$. thaliana target region}

We targeted the $222 \mathrm{~kb}$ region on A. thaliana chromosome 4 , which had been studied previously in $B$. rapa [30], B. oleracea [29] and B. napus [31] and is also partially duplicated on $A$. thaliana chromosome 5 , resulting from the known $\alpha$-duplication event. Seventeen probes representing $A$. thaliana genes from this region were hybridised to high-density colony filters of the BGH library. The probes identified 18-207 BACs each, with 207 BAC clones being recognised by two or more probes. The resulting 1,110 BAC clones were endsequenced, with successful sequence being acquired from 851 clones. These sequence data were compared against each other and against the A. thaliana gene complement using BLASTN, which identified 483 potentially non-redundant BAC clones for further analyses. The resultant banding patterns of the HindIII digested BAC clones hybridised with the gene specific probes were studied to confirm the initial data and to find those clones with shared loci (Additional file 1: Table S1 and Figure 1). Of the 483 selected BACs a total of 362 clones 


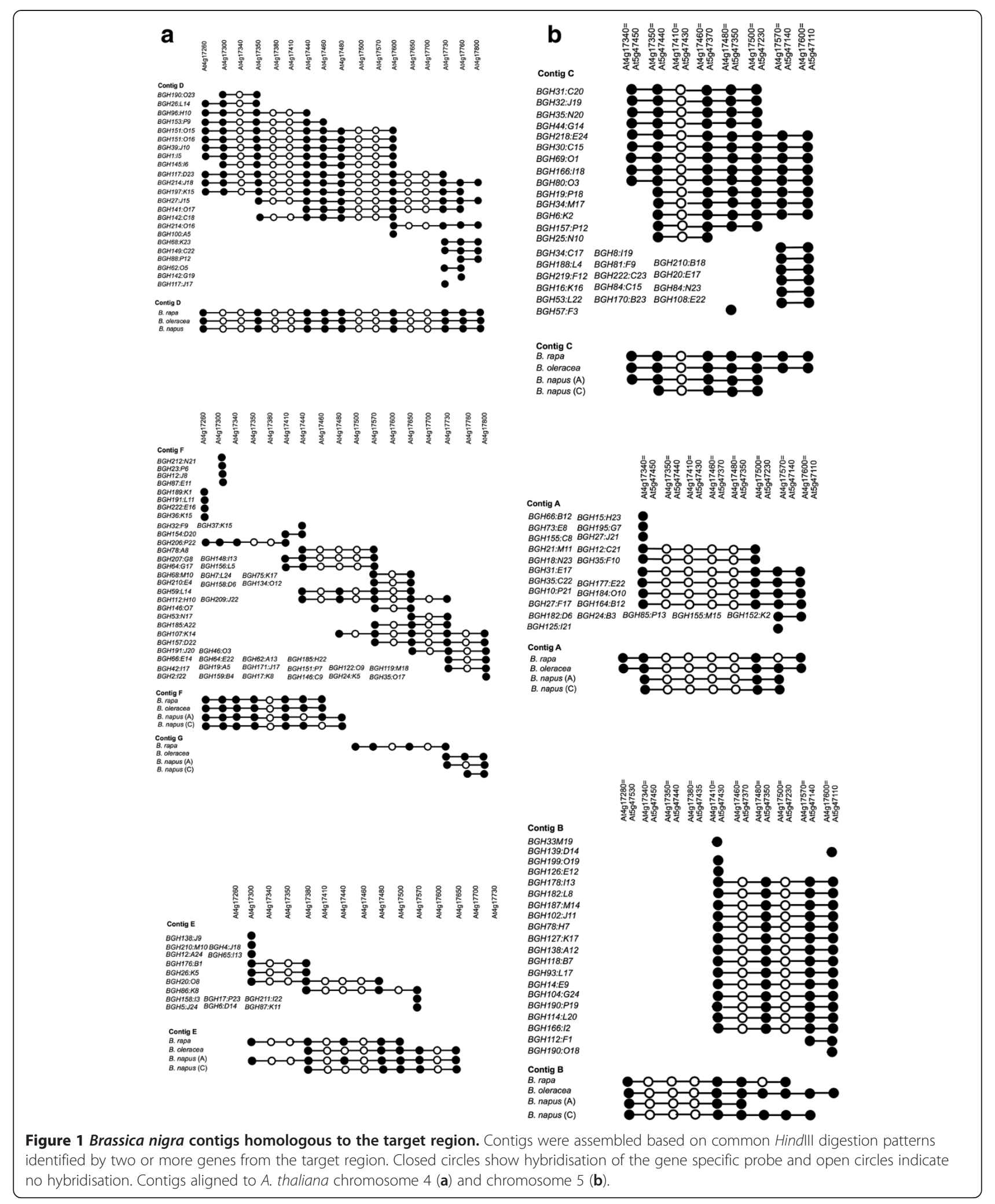


Table 1 Details of probes used to screen B. nigra BAC library and the results from the hybridisation data

\begin{tabular}{|c|c|c|c|c|}
\hline Gene specific probe & Old code & Number of positive clones & Number of loci & Mean redundancy \\
\hline At4g17260 & DL 4665 & 28 & 1 & 28.0 \\
\hline At4g 17300 & DL 4685 & 37 & 1 & 37.0 \\
\hline At4g17340 & DL 4705 & 77 & 1 & 77.0 \\
\hline At4g 17350 & DL 4710 & 54 & 2 & 27.0 \\
\hline At4g 17380 & DL 4725 & 17 & 2 & 8.5 \\
\hline At4g 17410 & DL 4740 & 43 & 2 & 21.5 \\
\hline At4g 17440 & DL 4755 & 83 & 1 & 83.0 \\
\hline At4g 17460 & DL 4765 & 51 & 3 & 17.0 \\
\hline At4g17480 & DL 4775 & 35 & 4 & 8.7 \\
\hline At4g17500 & DL 4785 & 40 & 1 & 40.0 \\
\hline At4g 17570 & DL 4820 & 58 & 2 & 29.0 \\
\hline At4g17600 & DL 4835 & 107 & 2 & 53.5 \\
\hline At4g 17650 & DL 4860 & 21 & 1 & 21.0 \\
\hline At4g17700 & DL 4885 & 10 & 3 & 3.3 \\
\hline At4g 17730 & DL 4900 & 67 & 1 & 67 \\
\hline At4g17760 & DL 4915 & 19 & 2 & 9.5 \\
\hline At4g 17800 & DL 4935 & 85 & 3 & 28.3 \\
\hline
\end{tabular}

showed strong positive hybridisation for the 17 genespecific probes while 121 from this subset did not. The number of BACs and loci identified for each gene specific probe is reported in Table 1, with the number of homologous loci ranging between 1 and 4 for each $A$. thaliana gene.

Contigs of overlapping BAC clones were assembled based on common digestion patterns observed for two or more genes. In addition, all 483 BAC clones were subjected to SNaPshot high information content fingerprinting (HICF) and the FPC software was used to assemble contigs $[38,41,42]$. In FPC it is recommended to start building contigs at high stringency to prevent chimeric joining of duplicated regions and to iteratively lower the stringency to avoid gaps in the resultant physical map [43]. Therefore we started with a cut off value of $1 \times 10^{-35}$ for automatic contig assembly and used the "DQer" function to break up Q contigs (contigs containing more than 10\% Questionable clones) which resulted in 16 contigs and $62 \%$ (302) singletons. The stringency was lowered in a stepwise manner and the project finished with an optimal lower cut off value of $1 \times 10^{-15}$, which resulted in 35 contigs with $38 \%$ (186) singletons that could not be incorporated into other contigs (Additional file 1: Table S1). This resulted in an assembly with a low number of $\mathrm{Q}$ clones ( $0-5$ clones in each contig). Six $\mathrm{B}$ genome contigs which had the highest number of overlapping BACs (Figure 1) and were in agreement with the Southern hybridisation results were considered representative of the A. thaliana target region. The assembled contigs appeared to follow the order of the genes as represented in the $A$. thaliana genome (Additional file 1: Table S2 and Figure 1). Three $\mathrm{B}$ genome regions were homologous to $A$. thaliana chromosome 5 (contigs A, B and C) while the other three were homologous to A. thaliana chromosome 4 (contigs, $\mathrm{D}, \mathrm{E}$ and $\mathrm{F}$ ), and the contigs were named accord to the previous analysis of the Brassica A/C genomes [29,30]. The contig assignments were based on the presence or absence of eight genes (At4g17260, At4g17300, At4g17380, At4g17440, At4g17650, At4g17750, At4g17760, and At4g17800) that are present in the chromosome 4 region, but not in the chromosome 5 region $[29,44]$. At this macro-level the only apparent major difference between the Brassica $\mathrm{B}$ genome and $\mathrm{A}$ and $\mathrm{C}$ genomes was the joining of contigs $\mathrm{F}$ and $\mathrm{G}$ in the $\mathrm{B}$ genome, which are physically separated in the A/C genomes (Figure 1).

\section{Comparative organisation at the sequence level}

BACs were selected for sequencing in order to represent each of the six contiguous regions identified as being homologous to the $A$. thaliana region (Figure 1 ). The list of BAC clones sequenced, and their characteristics are detailed in Table 2.

Orthologous genes between $B$. nigra and $A$. thaliana were identified using sequence similarity, each sequenced contig was searched against the $A$. thaliana gene sequences (TAIR 10) using BLASTN and in addition were verified using $a b$ initio prediction. The results are summarized in Figure 2 and show extensive conservation of gene content and order across each set of related genome segments.

Five examples of gene tandem duplication were identified in B. nigra compared to A. thaliana, At5g47150 in 
Table 2 List of Sequenced B. nigra BAC clones

\begin{tabular}{lllc}
\hline B. nigra BAC & Genome segment & Size bp & Accession number \\
\hline BGH184:O10 & B. nigra Contig A & 116,795 & KC96003 \\
BGH31:E17 & B. nigra Contig A & 257,762 & KC95996 \\
BGH93:L17 & B. nigra Contig B & 258,014 & KC96000 \\
BGH8:119 & B. nigra Contig C & 127,829 & KC95992 \\
BGH34:M17 & B. nigra Contig C & 158,918 & KC95997 \\
BGH24:O18 & B. nigra Contig D & 139,013 & KC95995 \\
BGH214:117 & B. nigra Contig D & 384,919 & KC96005 \\
BGH214:O16 & B. nigra Contig D & 140,361 & KC96006 \\
BGH20:O8 & B. nigra Contig E & 142,538 & KC95994 \\
BGH12:A24 & B. nigra Contig E & 121495 & KC95993 \\
BGH148:113 & B. nigra Contig F & 203,952 & KC96002 \\
BGH59:L14 & B. nigra Contig F & 169,401 & KC95998 \\
BGH64:E22 & B. nigra Contig F & 124,814 & KC95999 \\
BGH107:K14 & B. nigra Contig F & 170,106 & KC96001 \\
BGH206:P22 & B. nigra Contig F & 122,354 & KC96004 \\
\hline
\end{tabular}

contig A, At4g17650 and At4g17570 in contig F, and At4g17350 and At4g17600 in contig D (Figure 2). In the 6 contigs, 24 genomic insertions were detected which disrupted the collinearity (Figure 2), one of which was also a tandem repeat, At4g20140 in contig F. In five instances genes that were duplicated or triplicated in $A$. thaliana were present in only one copy in the B. nigra genome, apart from At5g47350 in B genome contig C where the homologous gene family was represented by two copies (Figure 2). The most significant microrearrangement that was specific to the $B$ genome was observed in contig $\mathrm{B}$ where the region containing At5g47480 and At5g47490 was duplicated and inverted (Figures 2 and 3). Most of these rearrangements were specific to one contig, and one genome, apart from the triplicated gene family of At4g17470, At4g17480 and At4g17483, which was represented by one gene in all three genomes [32,34]. Contig E in B. nigra showed the highest relative incidence of gene insertion or transposition with concomitant absence of conserved gene content, with one region, between At4g17380 and At4g17480, being disrupted by the presence of six genes from unrelated regions of the $A$. thaliana genome. The same region showed a similar level of discontinuity in the $\mathrm{C}$ genome but the $\mathrm{A}$ genome although missing conserved genes showed little evidence of gene insertion. The analysis at the sequence level demonstrated that unlike the $\mathrm{A}$ and $\mathrm{C}$ genomes where an inversion, as observed in the $B$. rapa genome sequence [45] has separated contigs $\mathrm{F}$ and $\mathrm{G}$, the $\mathrm{B}$ genome has maintained collinearity with A. thaliana chromosome 4. The set of genes from $A$. thaliana chromosome 2 at the top of contigs $\mathrm{A}$ and $\mathrm{B}$ of $B$. nigra indicate a rearrangement end point. This discontinuity in the collinearity was previously observed in B. rapa and indicates a break in the ancestral conserved block structure [46].

\section{Comparative alignment of homoeologous segments in Brassica diploid genomes}

The overall similarity of each of the homoeologous regions of the three diploid genomes, A, B and C was compared at the nucleotide level using MUMmer as shown in Figure 3 [47]. In general as expected from the observed gene content the six regions show good conservation between the three genomes. However, the sequence alignments uncover instances of genome duplications, deletions/expansions and inversions that are specific to each of the three genomes. Contigs $\mathrm{E}$ and $\mathrm{F}$ show the most distinct differences. Contig E contains a large number of genes from non-collinear regions of the genome and in addition shows further expansion through repeat element proliferation, interestingly the pattern of genome expansion is conserved between the $\mathrm{B}$ and $\mathrm{C}$ genomes; however, the types of genetic elements represented is not (Figure 2). Contig $\mathrm{F}$ has seen expansion in the $\mathrm{C}$ genome through tandem duplication of members of a gene family of proline rich genes. The $\mathrm{C}$ genome has more observed instances of genome expansion compared to either the A or B genomes, which is particularly notable in contig B (Figure 3 and Table 3 ). The remarkable expansion of this region in the $C$ genome is due to the insertion of a large non-collinear segment ( 200 kb) between At5g47070 and At5g47100.

\section{Level of gene conservation and fractionation in triplicated regions}

As with previous comparative analysis conservation was inferred from the presence of significant sequence similarity between genic regions in the three genomes. However, this does not extend to the maintenance of entire gene structures and fractionated exon loss is a common feature of plant genome evolution [48]. The retention of exonic regions for the conserved $A$. thaliana genes was calculated for each Brassica genome contig (Table 3 and Additional file 1: Table S3). The results as shown in Table 3 indicate that for five out of the six regions the B genome has maintained a higher number of complete gene copies. For those B genome contigs homologous to A. thaliana chromosome 4 , two of the regions have a lower level of fractionation compared to the third (74\% retention of exonic regions in contigs $\mathrm{F}$ and $\mathrm{D}, 38 \%$ in contig E). While the $\mathrm{A}$ and $\mathrm{C}$ genomes show a similar trend, the expected stronger conservation of one of the three duplicated regions is more apparent. The two triplicated regions of the B genome studied follow the pattern of genome maintenance as observed previously for the A genome where one region, the 'least fractionated' 


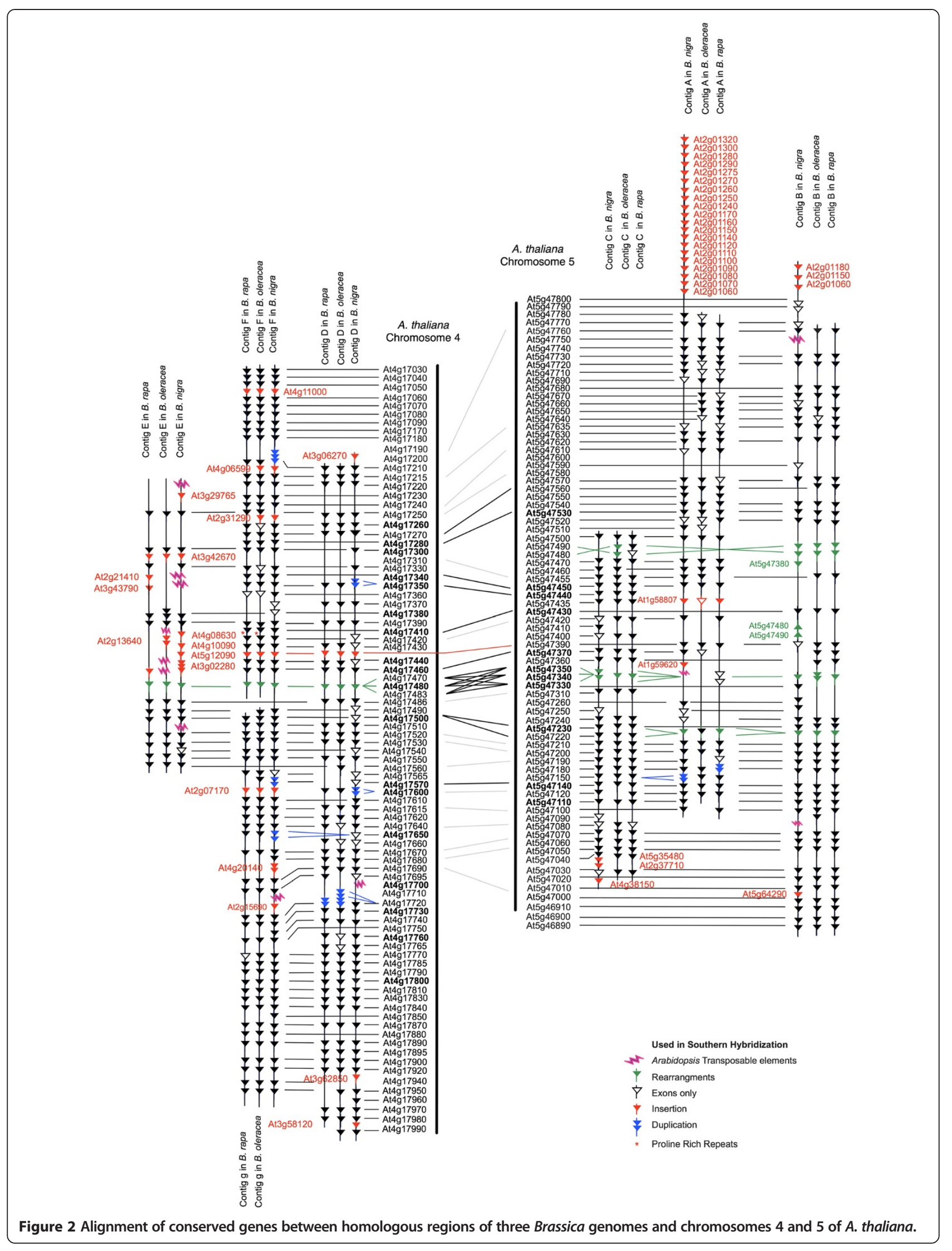



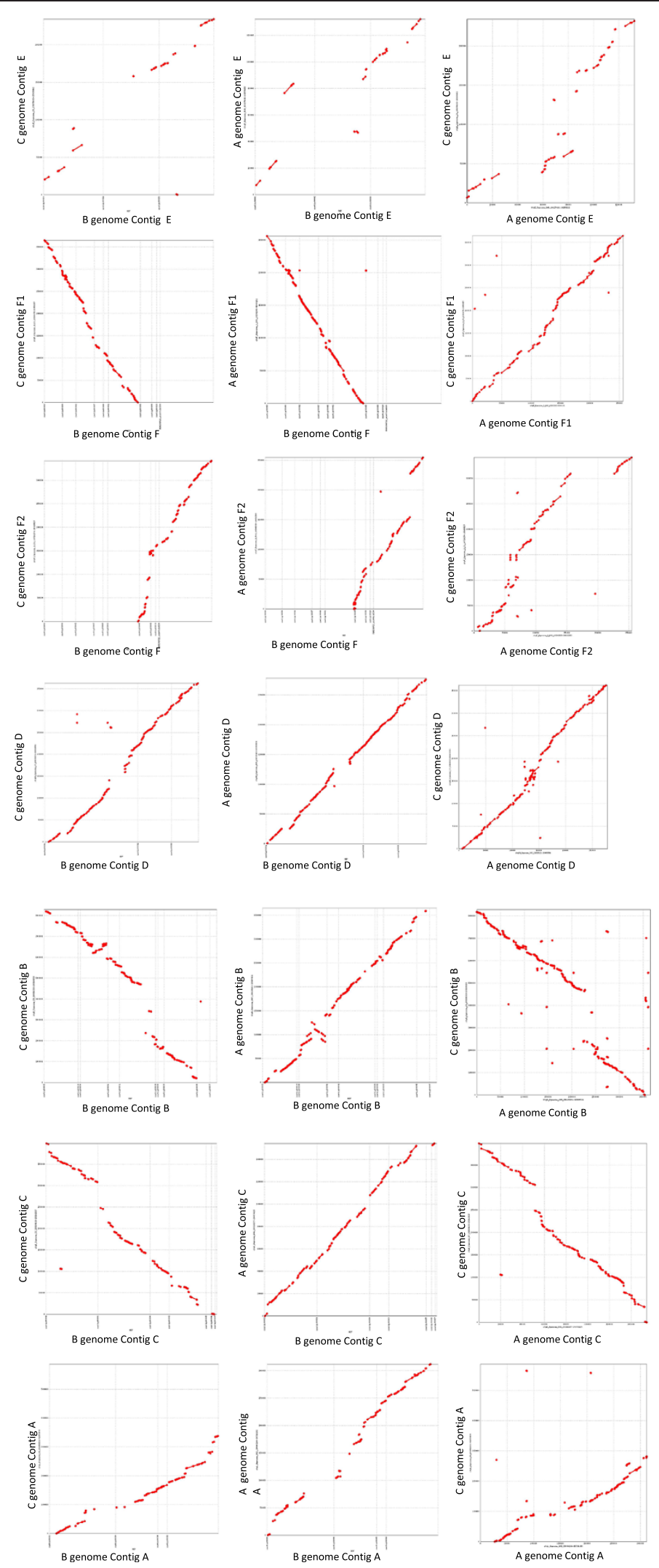

Figure 3 Alignment of $B$. nigra contigs with their homologous segments in the A and $C$ genomes as found by MUMmer. 
Table 3 Level of fractionation in the triplicated regions of the three Brassica genomes

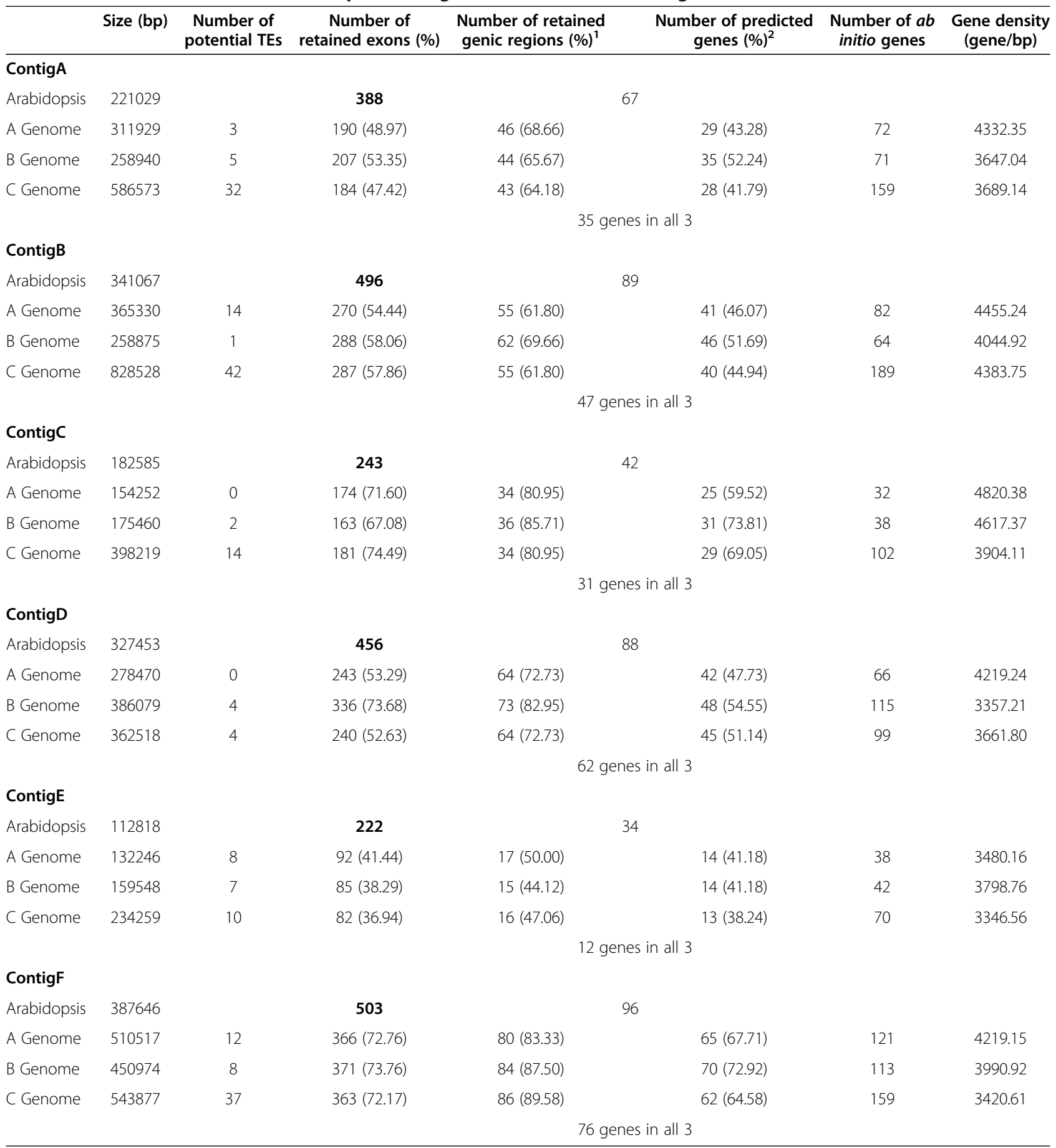

1. Indicates the presence of complete and partial genes as determined by significant homology to any region of the annotated genes in $A$. thaliana. 2. Indicates homology to complete $A$. thaliana genes based on the maintenance of intron/exon structure.

(LF) is more highly conserved than either of the other two, 'more fractionated1' (MF1) and MF2 [45]. However, the two MF regions of the $\mathrm{B}$ genome appear to have incurred reduced deletion events compared to the $\mathrm{A}$ and $\mathrm{C}$ genomes as shown by a higher prevalence of gene retention (Table 3 and Additional file 1: Table S3). It has been suggested previously that higher levels of fractionation can be the result of on-going transposable element activity [49]; however, although in general the $C$ genome shows both an expansion in genome size and repetitive element composition, this is not true for the A genome (Table 3). 
Timing of genome divergence

Synonymous base substitution rates (Ks values) were calculated for variable numbers of conserved genes across each genomic region of the three Brassica genomes and A. thaliana (Table 4). The mean values of Ks and divergence times are in agreement with values reported previously for the A and $\mathrm{C}$ genomes [25,33]. The divergence time of the ancestral Brassica genome from A. thaliana was estimated between 13.9 Mya in contig A to $\sim 17$ Mya in contig D, which is similar to previous estimates of 14.5 - 20 Mya [24]. Contigs homologous to $A$. thaliana chromosome 4 in all three Brassica genomes were suggested to be significantly older than those homologous to chromosome 5 ( $\mathrm{p}<0.0001$ ), which could suggest that as in many plant species the whole genome duplication event in A. thaliana was as a result of allopolyploidy. Previous estimates suggest $B$. rapa and $B$. oleracea diverged at around 3.7 Mya [26] and our calculations are in accordance with an average value of 3.2 Mya. The divergence time of $B$. nigra from the $B$. rapa/ oleracea lineage was estimated previously based on limited available sequence data to be 7.9 Mya [25]. As detailed in Table 3 the calculated divergence times for $B$. nigra from $B$. rapa/oleracea for the target regions varied with an average of 6.2 Mya, although lower values were observed for contig $\mathrm{E}$ that could result from a smaller number of genes being available for comparison. It was assumed that the whole genome triplication (WGT) event observed in the $\mathrm{B}$ genome was shared with the $\mathrm{A}$ and $\mathrm{C}$ genomes and calculation of $\mathrm{Ks}$ values for conserved genes across genomic segments within each of the Brassica genomes corroborated this assumption, with no significant difference between the average age of divergence of the WGT $(11.6 \pm 3.4$ Mya).

\section{Discussion}

In the 1950's when B. juncea replaced B. nigra as the mustard crop of choice in Asia, B. nigra was effectively abandoned with regards to crop improvement [50] and has been the subject of limited breeding, genetics and genomics research compared to the other two diploid species of U's triangle [51]. However, the Brassica B genome has been recognised as a useful source of novel alleles for various traits of interest, in particular disease resistance and tolerance to abiotic stress, and numerous attempts have been made to transfer these traits to the Brassica A and $C$ genomes, with varying success $[9,13,14,17,52-54]$. An improved understanding of the genome structure and gene composition of the Brassica $\mathrm{B}$ genome could provide insights into its relationship among the Brassica species and could potentially facilitate exploitation of this important resource. The current study benefited from previous research that had studied extensively the organisation of the Brassica $\mathrm{A}$ and $\mathrm{C}$ genome regions homologous to a $222 \mathrm{~kb}$ region of $A$. thaliana chromosome four, which was itself duplicated on chromosome five [44].

Table 4 Pair wise divergence time of genome segments based on synonymous base substitution rates

\begin{tabular}{|c|c|c|c|c|c|c|c|}
\hline \multirow[t]{2}{*}{ Region } & \multirow[b]{2}{*}{ Number of genes } & \multicolumn{2}{|l|}{ A genome } & \multicolumn{2}{|l|}{ C Genome } & \multicolumn{2}{|l|}{ Arabidopsis } \\
\hline & & Average Ks & Mya & Average Ks & Mya & Average Ks & Mya \\
\hline B genome Contig A & 15 genes & $0.18 \pm 0.07$ & $6.073 \pm 2.18$ & $0.19 \pm 0.06$ & $6.60 \pm 2.04$ & $0.42 \pm 0.10$ & $14.30 \pm 3.28$ \\
\hline A genome Contig A & & & & $0.09 \pm 0.05$ & $3.22 \pm 1.68$ & $0.41 \pm 0.09$ & $13.68 \pm 2.91$ \\
\hline$C$ genome Contig $\mathrm{A}$ & & & & & & $0.41 \pm 0.09$ & $13.96 \pm 3.16$ \\
\hline B genome Contig B & 25 genes & $0.20 \pm 0.07$ & $6.92 \pm 2.25$ & $0.22 \pm 0.06$ & $7.36 \pm 2.10$ & $0.42 \pm 0.09$ & $14.20 \pm 2.99$ \\
\hline A genome Contig B & & & & $0.09 \pm 0.05$ & $3.20 \pm 1.65$ & $0.43 \pm 0.10$ & $14.44 \pm 3.24$ \\
\hline C genome Contig B & & & & & & $0.45 \pm 0.10$ & $15.04 \pm 3.31$ \\
\hline B genome Contig C & 16 genes & $0.18 \pm 0.06$ & $6.30 \pm 2.01$ & $0.18 \pm 0.07$ & $6.06 \pm 2.41$ & $0.45 \pm 0.13$ & $15.07 \pm 4.45$ \\
\hline A genome Contig C & & & & $0.10 \pm 0.06$ & $3.47 \pm 1.85$ & $0.43 \pm 0.10$ & $14.54 \pm 3.41$ \\
\hline C genome Contig C & & & & & & $0.43 \pm 0.11$ & $14.39 \pm 3.61$ \\
\hline B genome Contig D & 28genes & $0.20 \pm 0.11$ & $6.81 \pm 3.67$ & $0.19 \pm 0.09$ & $6.34 \pm 2.96$ & $0.50 \pm 0.14$ & $16.69 \pm 4.69$ \\
\hline A genome Contig $D$ & & & & $0.10 \pm 0.06$ & $3.59 \pm 1.85$ & $0.52 \pm 0.18$ & $17.40 \pm 5.86$ \\
\hline$C$ genome Contig D & & & & & & $0.50 \pm 0.18$ & $16.99 \pm 5.99$ \\
\hline B genome Contig E & 7 genes & $0.15 \pm 0.04$ & $5.17 \pm 1.46$ & $0.14 \pm 0.04$ & $4.91 \pm 1.38$ & $0.48 \pm 0.19$ & $16.03 \pm 6.44$ \\
\hline A genome Contig E & & & & $0.08 \pm 0.05$ & $2.97 \pm 1.51$ & $0.46 \pm 0.18$ & $15.47 \pm 6.12$ \\
\hline$C$ genome Contig $\mathrm{E}$ & & & & & & $0.46 \pm 0.18$ & $15.59 \pm 6.12$ \\
\hline B genome Contig F & 41 genes & $0.19 \pm 0.05$ & $6.46 \pm 1.91$ & $0.18 \pm 0.06$ & $6.32 \pm 1.98$ & $0.47 \pm 0.14$ & $15.93 \pm 4.70$ \\
\hline A genome Contig $F$ & & & & $0.08 \pm 0.03$ & $2.76 \pm 1.11$ & $0.48 \pm 0.14$ & $16.10 \pm 4.64$ \\
\hline$C$ genome Contig $\mathrm{F}$ & & & & & & $0.47 \pm 0.14$ & $15.83 \pm 4.65$ \\
\hline
\end{tabular}


The Brassica B genome was separated previously from the $\mathrm{A} / \mathrm{C}$ genome lineage in phylogenetic analyses $[23,55]$. Analysis of the six regions within the Brassica B genome compared to both the $\mathrm{A}$ and $\mathrm{C}$ genomes allowed a more accurate estimate of the timing for divergence of the two lineages, $\sim 6.2$ Mya $( \pm 2.19)$, compared to those previously published which ranged widely from 7.9-14.6 Mya [25] to 5-10 Mya [56]. The pattern of genome rearrangements and gene deletions that differentiate the Brassica genomes over this period have led to the extant species. The Brassica B genome as for the other diploid Brassica genomes, retained three genomic copies of each $A$. thaliana region, reflecting the underlying whole genome triplication (WGT) event that distinguishes the Brassiceae tribe [25]. The same rate of synonymous substitution was observed between the triplicated copies of the three Brassica genomes and $A$. thaliana (Table 4) and similarly the intragenomic rate, (A: $\mathrm{Ks}=0.323 \pm 0.12, \mathrm{~B}: \mathrm{Ks}=0.353 \pm 0.08, \mathrm{C}: \mathrm{Ks}=0.353 \pm$ 0.11 ) across the triplicated regions within the genomes was relatively equivalent, suggesting the $\mathrm{B}$ genome evolved from the same paleohexaploidy event as the A and $\mathrm{C}$ genome. As reported previously in B. oleracea, $B$. rapa and $B$. napus [32,34] and shown in Figure 2 there is a high level of conservation for the composition and order of genes among the three Brassica diploid genomes compared to $A$. thaliana. Observed breaks in collinearity were due to predominantly minor rearrangements, such as inversions, duplications, small insertions and deletions, although one large insertion (> $200 \mathrm{~kb}$ ) of a non-collinear segment in the $\mathrm{C}$ genome was found. One major chromosomal rearrangement, a large inversion relative to $A$. thaliana chromosome four, differentiated the A/C lineage from the Brassica B genome for one of the triplicated copies, which was also apparent from the genome sequence of $B$. rapa [45]. Genetic mapping of the B genome in $B$. juncea has certainly indicated that a number of large-scale chromosomal rearrangements may differentiate the Brassica A and B genomes [22], although this study also indicated that at least three of the $\mathrm{B}$ genome chromosomes were virtually collinear with their A genome homoeologue [22]. In addition, they identified conservation of ancestral Brassicaceae block structures across the three diploid Brassica genomes. Interestingly, as indicated in Figure 2, endpoints delineating ancestral Brassicaceae blocks, in this case between $A$. thaliana chromosomes two (block K) and five (block V), were found to be conserved between at least two of the triplicated copies in both the Brassica A (on linkage groups A02 - contig L, and A09 - contig B) and $B$ genomes $[45,46]$. The identification of such conserved ancestral rearrangements will further assist in defining the structure of the progenitor genome of the Brassiceae tribe, which is proposed to differ from that of A. thaliana [57].
Although the regions studied demonstrated marked conservation relative to $A$. thaliana, each species and each genomic region were characterized by the presence of gene sequences from non-collinear regions, which were rarely shared. However, there appear to be regions that are more sensitive to such alterations and although they are common to all genomes in lacking collinearity (for example in contig E between At4g17480 and At4g17390) the changes observed were specific to each. In addition, each of the regions across the genomes varied in size (Table 3 ). The B. nigra B genome has been reported to be $632 \mathrm{Mb}(0.65 \mathrm{pg})$, smaller than $B$. oleracea at $696 \mathrm{Mb}(0.71 \mathrm{pg})$ but larger than $B$. rapa at $529 \mathrm{Mb}$ (0.54 pg), (Johnston et al., 2004). It had previously been shown that the $\mathrm{C}$ genome was expanded compared to the A genome for the targeted regions, which would be in accordance with current genome estimates; however, our estimates for this region place the $\mathrm{B}$ genome closer in size to that of $B$ rapa.

There has been considerable interest in attempting to transfer genomic regions from the $\mathrm{B}$ genome into the $\mathrm{A}$ and $C$ genomes of $B$. napus $[4,13,14,58]$; however, this has been largely unproductive due to limited or no recombination occurring between introduced $\mathrm{B}$ genome chromosomes and their supposed orthologues. Even the use of artificial resynthesis to develop new allopolyploids, which greatly enhances the level of recombination between the Brassica A and $\mathrm{C}$ genomes [11], has been ineffectual [59]. Yet at the genetic level [22] and as shown here at the sequence level there is marked conservation across the three Brassica genomes, in fact the level of karyotype and microstructure divergence across the three genomes is comparable. The question is why is aberrant homoeologous recombination so frequently observed in newly resynthesized $B$. napus (approximately $10 \%$ of the total observed recombination) but absent in equivalent resynthesized B. juncea [11,59]. Although it is possible that there is a genetic barrier to recombination such as that found in wheat [60] or proposed for $B$. napus [18] the inability to breakdown this control would suggest limited or no variation exists for the trait, or unlike the progenitors of wheat the $\mathrm{B}$ genome diploid maintains activity for a trait which has value only in the polyploid state. If the barrier is then physical, then chromosomal rearrangements not captured in previous genetic mapping studies presumably differentiate the genomes, one such example would be the inversion found here that is specific to the $\mathrm{A} / \mathrm{C}$ lineage. Inversions have been proposed to have a significant impact on plant evolution [61] and could have lead to the reproductive isolation and speciation of the $B$ genome.

Although the current analyses focuses on two triplicated regions of the Brassica B genome, it provides insights into the gene retention pattern across the wider 
genome, since the A genome regions studied here reflect the pattern found in the B. rapa genome sequence [45]. The publication of the first Brassica genome sequence, that of $B$. rapa, provided extensive evidence of gene fractionation following the WGT and perhaps most interestingly due to bias in gene retention in the three genomic copies led to the two-step polyploidy hypothesis with fractionation between steps [45]. This hypothesis, in which the paleohexaploid was formed from the hybridisation between a diploid and an established tetraploid, has been rigorously tested since, with the retention of exonic regions being studied in detail for the A genome [62] and indicating that deletions are the major mode of gene inactivation and hence evolutionary drive in Brassica species. For the studied regions, the B genome appears to follow the same gene fractionation pattern as the A genome with one of the three genomic copies showing a higher level of conservation (Figure 4; Additional file 1: Table S3). The observed level of gene loss or fractionation for the triplicated regions at each important branch point in the evolution of the studied genomes is indicated in Figure 4. The highest percentage of fractionation appears to have occurred prior to the separation of the three genomes that is where gene loss is shared across all three genomes. Although the general pattern of fractionation holds true species-specific differences can be created through the types of genes that are maintained. For example in the regions homologous to A. thaliana chromosome five, no copies of two annotated disease resistance genes, At5g47250 and At5g47260, are found in the A and C genomes, yet multiple full or partial copies are maintained for each in the three homologous B genome regions. In addition, local insertion of a further annotated disease resistance gene (At1g59620) in this region of the B genome for contig A indicates a resistance cluster specific to the $\mathrm{B}$ genome.

\section{Conclusions}

Analysis of homologous regions of three closely related Brassica diploid genomes has identified extensive conservation of gene content and order, which is not reflected in the established phylogenetic relationship of the three species. The genome of B. nigra, based on its inability to establish effective pairing structures with the $\mathrm{A}$ and $\mathrm{C}$ genomes, might have been expected to have undergone more rearrangements at the micro-level, suggesting perhaps that major chromosomal events such as the observed inversion could have played a significant role in the speciation of the $\mathrm{B}$ genome. The $\mathrm{B}$ genome is likely to be an excellent source of novel genes for a number of important traits and capturing this diversity through genome sequencing could prove important for future crop improvement.

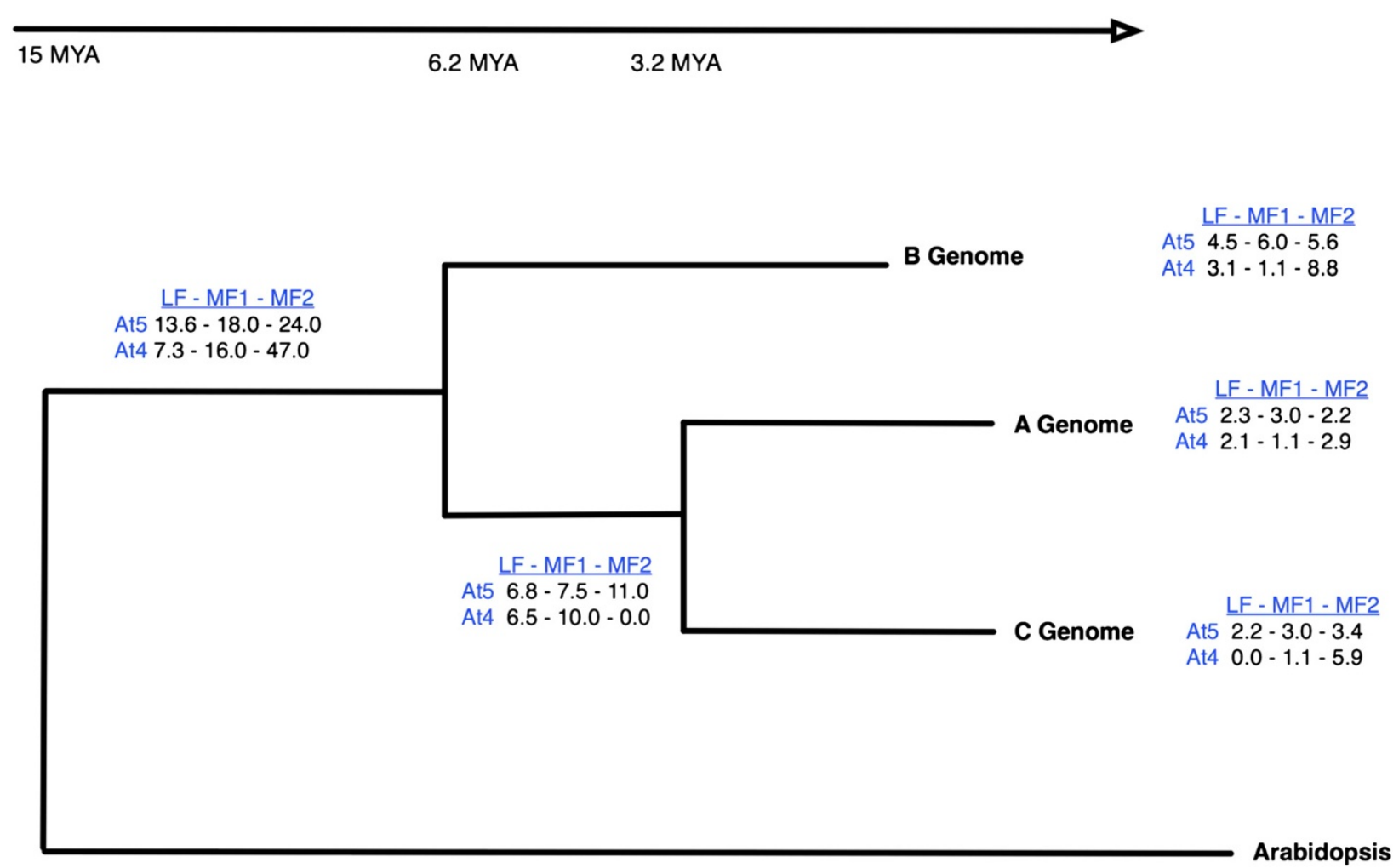

Figure 4 Phylogenetic tree of the Brassica B and A/C lineage. The percentage of fractionation for the three sub-genomes at each node is specified. The contigs are ordered according to the overall level of fractionation from least (LF) to most fractionated (MF2) (Table 3). 


\section{Methods}

\section{Construction of BAC library}

Seedlings of a doubled haploid B. nigra (DH No100, provided by Alison Ferrie, National Research Council of Canada Plant Biotechnology Institute (NRC-PBI), Saskatoon) were grown in a controlled environment cabinet at a constant $20^{\circ} \mathrm{C}$ with 16 hour photoperiod until 6-8 leaf stage. Plants were placed in the dark for 3 days prior to tissue harvest, leaf tissue was flash frozen in liquid nitrogen and shipped to BioS\&T, Montreal, Canada (http://www. biost.com/) for library construction. Large insert genomic DNA was ligated into the HindIII site of the pIndigoBAC5 vector (Epicentre, Madison, WI, US). The library clones are prefixed "BGH" and are arrayed in 222384 well plates, with an average insert size of $130 \mathrm{~kb}$, representing almost a $20 \mathrm{X}$ coverage of the Brassica B genome which has an estimated size of $632 \mathrm{Mb}$ based on flow cytometry [40].

\section{Primary BAC library screening}

The library was gridded in replicate on five $22.2 \mathrm{~cm}^{2}$ Hybond $^{\mathrm{Tm}}$-XL (GE Healthcare Uppsala, Sweden) membranes and screened by colony hybridisation using probes of seventeen genes from the A. thaliana $222 \mathrm{~kb}$ target region of chromosome 4 [29] to identify homologous clones (Table 1). Preparation of the $A$. thaliana gene specific probes and their sequence are detailed in O'Neill and Bancroft (2000). These probes were labeled by the random priming method using the RediPrime II kit (GE Healthcare Uppsala, Sweden) according to the manufacturer's instructions. Hybridisation was carried out at $65^{\circ} \mathrm{C}$ for $16 \mathrm{~h}$ in QuikHyb Hybridisation Buffer (Agilent Technologies Santa Clara, CA) according to the manufacturer's instructions. The membranes were washed twice for $10 \mathrm{~min}$ at $65^{\circ} \mathrm{C}$ followed by three washes for $10 \mathrm{~min}$ at room temperature in $2 \times \mathrm{SSC}$ and $0.1 \% \mathrm{SDS}$.

\section{BAC DNA preparation}

DNA from BAC clones identified in the primary screen was prepared in one of two ways: for DNA digestion, HICF and BAC end sequencing, BAC DNA was prepared using standard alkaline lysis [63]. For full length sequencing, DNA was isolated using the Qiagen Large Construct Kit (Qiagen, Valencia, CA) according to the manufacturer's instructions. The integrity and size of the clones was confirmed by digestion with NotI and resolution by Pulse Field Gel Electrophoresis (PFGE) on a $1 \%$ agarose gel in $0.5 \times \mathrm{TBE}$ at $120^{\circ}$ angle for 16 hours at $14^{\circ} \mathrm{C}$ with a $0.1-40.0$ second switch. The sizes of the fragments were estimated using the Lambda Ladder PFG and MidRange II PFG markers (NEB Ipswich, MA).

\section{Southern blot analysis}

Purified BAC DNA was digested with HindIII enzyme and separated on $1 \%$ agarose in $1 \times$ TAE at $5 \mathrm{~V}$ for
18 hours. The digested DNA was blotted onto Hybond $\mathrm{XL}$ membranes and probed as above. Hybridisation was done at $65^{\circ} \mathrm{C}$ for $16 \mathrm{~h}$ in modified Church buffer [64] and the membranes were washed twice for $10 \mathrm{~min}$ at room temperature in $2 \times \mathrm{SSC}$ and $0.1 \%$ SDS.

\section{BAC sequence analyses}

BAC end sequencing was completed for 1,110 positive clones using modified BigDye Terminator v.3.1 and BigDye Xterminator purification kit protocols (Life Technologies Carlsbad, CA). Sequencing reactions were run on an AB $3730 \times 1$ at the NRC DNA Technologies Laboratory in Saskatoon. The resultant sequence data was compared to itself and to A. thaliana using BLASTN with default parameters and an E value cut-off of $1 \times \mathrm{E}$ 10. Full length BAC sequences were generated with Roche 454 Flex sequencing also at NRC. The 15 BACs were each indexed and sequenced in one half of a 454 plate. The sequences were assembled into large contigs using Newbler v 2.6 (Roche Diagnostics).

\section{SNaPshot fingerprinting}

The fingerprinting reaction was performed following Luo et al., (2003) with minor modifications recommended by the Arizona Genomics Institute. The BAC DNA was incubated with the digestion and labeling mix for one hour at $37^{\circ} \mathrm{C}$ and labelling was performed for one hour at $65^{\circ} \mathrm{C}$. The size standard geneScan LIZ-1200 (size range from 20 to $1200 \mathrm{bp}$ ) was added to each sample prior to loading on an AB 3100 for capillary electrophoresis using the DS-02 dye set. Peak height, area and sizes were collected by GeneMapper ${ }^{\circledR}$ software v3.7 and converted into FPC readable format after editing by the GenoProfiler ${ }^{\circledR}$ v2.1 (http://wheat.pw.usda.gov/PhysicalMapping/; [41]).

\section{Data editing operations and FPC contig assembly}

The editing process included removal of vector bands and removal of clones for which the reaction failed, lacked an insert or were suggestive of cross contamination [65]. The files generated by GenoProfiler were transferred to FPC v9.3 (www.agcol.arizona.edu/software/fpc; [42]). Initially, a tolerance value of 3.0 and a Sulston cut off score of $1 \times 10^{-35}$ was used to assemble contigs automatically, the stringency was later reduced as described in the Results section. "DQer" function of FPC was used to reassemble contigs with more than 10 $\mathrm{Q}$ clones. The resulting contigs were merged by the "End to End" auto merge function with a minimum of two matching ends. The remaining singletons were merged to form contigs by the "Auto merge/add" function. The fingerprints of clones in the merged contigs were then analysed manually and compared to contigs assembled based on Southern blot analyses to finalize the contigs [31]. 


\section{Sequence analyses}

The genome assembly of $B$. rapa (http://brassicadb.org) and preliminary assembly of $B$. oleracea (TO1000 sequencing consortium, unpublished data) were used to identify extended $\mathrm{A}$ and $\mathrm{C}$ genomic regions equivalent to the sequenced $\mathrm{B}$ genome regions. The $A$. thaliana genome sequence (TAIR-10; www.arabidopsis.org) was compared to the Brassica sequences using BLASTN with default parameters and an Expect value of $1 \times E^{-10}$. GlimmerHMM [66] was used to predict genes using training data from A. thaliana. MUMer and NUCmer were used to align contigs of the $\mathrm{AC}$ genome with those of the $\mathrm{B}$ genome and the alignments were visualised using MUMmer plot [47].

\section{Calculation of Ks values and phylogenetic tree}

Homologous predicted cDNA sequences from the Brassica genomes and $A$. thaliana were aligned using ClustalW version 1.83 [67]. Ks and Ka values were calculated in a pair wise model using the CODEML program in the PAML package version 4.4 [68]. The commonly adopted estimate of mutation rate of $1.5 \times 10^{-8}$ synonymous substitutions per site per year [69] was used to estimate the divergence time of the three genomes.

The alignments of the four orthologous gene sets were trimmed for regions with gaps and missing data using trimAl [70]. The alignment of 126 gene sets were concatenated using the Phyutility software [71], building a total alignment length of 120,936 bps which was then used for the phylogenetic analysis performed by the FastTree2.1.3 [72] and processed using Dendroscope [73]. Robustness of phylogenetic inference was assessed by the bootstrap analysis approach for 1000 reassembled alignments.

\section{Additional file}

Additional file 1: Table S1. Overlapping BACs based on Southern Hybridization analysis. +: positive hybridization with the gene specific probe, -: no hybridization. Highlighted cells are the fully sequenced BACs. Table S2. Overlapping BACs based on the FPC analysis. Table S3. Comparison of the level of exon retention across the three Brassica genomes.

\section{Competing interests}

The authors declare that they have no competing interests.

\section{Authors' contribution}

ZKN performed the research and analysed the data. ZKN and IAP designed the research and wrote the manuscript. TH prepared, quality assessed and carried out initial screening of the BAC library. IB and CMO provided the Arabidopsis probes and edited the manuscript. AGS assisted with sequencing of the BAC clones. All authors read and approved the final manuscript.

\section{Acknowledgments}

We like to thank members of Parkin lab, the late Dr. Steven Karcz, Mathew Links and Dr Sateesh Kagale at the AAFC Saskatoon, for their kind assistance.
The funding of this project was supported in part by the Canadian Brassica Genome Sequencing Project and by AAFC. We also like to thank Kristie de Baynast at the Arizona Genomics Institute and Dr. Ming-Cheng Luo at the UC Davis who helped us with fine adjustments of the SNaPshot protocol. Accession numbers: KC795992- KC796006.

\section{Author details}

${ }^{1}$ Agriculture and Agri-Food Canada, 107 Science Place, Saskatoon, SK S7N 0X2, Canada. ${ }^{2}$ DNA Technologies Laboratory, 110 Gymnasium Place, Saskatoon, SK S7N OW9, Canada. ${ }^{3}$ John Innes Centre, Norwich Research Park, Colney, Norwich NR4 7UH, UK

Received: 9 January 2013 Accepted: 4 April 2013

Published: 15 April 2013

\section{References}

1. Pradhan A, Nelson MN, Plummer JA, Cowling WA, Yan G: Characterization of Brassica nigra collections using simple sequence repeat markers reveals distinct groups associated with geographical location, and frequent mislabelling of species identity. Genome 2011, 54(1):50-63.

2. Negi MS, Sabharwal V, Bhat SR, Lakshmikumaran M: Utility of AFLP markers for the assessment of genetic diversity within Brassica nigra germplasm. Plant Breeding 2004, 123(1):13-16.

3. $U$ N: Genome analysis in Brassica with special reference to the experimental formation of $B$. napus and particular mode of fertilisation. Jap J of Bot 1935, 98:157-161.

4. Chevre AM, Eber F, Barret P, Dupuy P, Brace J: Identification of the different Brassica nigra chromosomes from both sets of B. oleracea-B. nigra and B. napus-B. nigra addition lines with a special emphasis on chromosome transmission and self-incompatibility. Theor Appl Genet 1997, 94(5):603-611.

5. Chevre AM, This P, Eber F, Deschamps M, Renard M, Delseny M, Quiros CF: Characterization of disomic addition lines Brassica napus-Brassica nigra by isozyme, fatty acid, and RFLP markers. Theor Appl Genet 1991, 81(1):43-49.

6. Sacristan MD, Gerdemann M: Different behavior of Brassica juncea and $B$. carinata as Sources of Phoma lingam resistance in experiments of interspecific transfer to B. napus. Plant Breeding 1986, 97:304-314.

7. Sjodin C, Glimelius K: Transfer of resistance against Phoma lingam to Brassica napus by asymmetric somatic hybridization combined with toxin selection. Theor Appl Genet 1989, 78(4):513-520.

8. Roy NN: Interspecific transfer of Brassica juncea-type high blackleg resistance to Brassica napus. Euphytica 1984, 33(2):295-303.

9. Struss D, Bellin U, Robbelen G: Development of B-genome chromosome addition lines of $B$. napus using different interspecific Brassica hybrids. Plant Breeding 1991, 106(3):209-214

10. Leflon M, Eber F, Letanneur JC, Chelysheva L, Coriton O, Huteau V, Ryder $C D$, Barker $G$, Jenczewski E, Chevre AM: Pairing and recombination at meiosis of Brassica rapa (AA) x Brassica napus (AACC) hybrids. Theor Appl Genet 2006, 113(8):1467-1480.

11. Parkin IA, Sharpe AG, Keith DJ, Lydiate DJ: Identification of the A and C genomes of amphidiploid Brassica napus (oilseed rape). Genome 1995, 38(6):1122-1131.

12. Sharpe AG, Parkin IA, Keith DJ, Lydiate DJ: Frequent nonreciprocal translocations in the amphidiploid genome of oilseed rape (Brassica napus). Genome 1995, 38(6):1112-1121.

13. Navabi ZK, Stead KE, Pires JC, Xiong Z, Sharpe AG, Parkin IA, Rahman MH, Good AG: Analysis of B-genome chromosome introgression in interspecific hybrids of Brassica napus x B. carinata. Genetics 2011, 187(3): 659-673.

14. Navabi ZK, Parkin IA, Pires JC, Xiong Z, Thiagarajah MR, Good AG, Rahman $\mathrm{MH}$ : Introgression of B-genome chromosomes in a doubled haploid population of Brassica napus x B. carinata. Genome 2010, 53(8):619-629.

15. Mason AS, Huteau V, Eber F, Coriton O, Yan G, Nelson MN, Cowling WA, Chevre AM: Genome structure affects the rate of autosyndesis and allosyndesis in $\mathrm{AABC}, \mathrm{BBAC}$ and $\mathrm{CCAB}$ Brassica interspecific hybrids. Chromosome research: an international journal on the molecular, supramolecular and evolutionary aspects of chromosome biology 2010, 18(6):655-666.

16. Mason AS, Nelson MN, Castello MC, Yan G, Cowling WA: Genotypic effects on the frequency of homoeologous and homologous recombination in 
Brassica napus x B. carinata hybrids. Theor App/ Genet 2011, 122(3):543-553.

17. Schelfhout CJ, Snowdon R, Cowling WA, Wroth JM: Tracing B-genome chromatin in Brassica napus x B. juncea interspecific progeny. Genome 2006, 49(11):1490-1497.

18. Jenczewski E, Eber F, Grimaud A, Huet S, Lucas MO, Monod H, Chevre AM: PrBn, a major gene controlling homeologous pairing in oilseed rape (Brassica napus) haploids. Genetics 2003, 164(2):645-653.

19. Liu Z, Adamczyk K, Manzanares-Dauleux M, Eber F, Lucas MO, Delourme R, Chevre AM, Jenczewski E: Mapping PrBn and other quantitative trait loci responsible for the control of homeologous chromosome pairing in oilseed rape (Brassica napus L.) haploids. Genetics 2006, 174(3):1583-1596.

20. Nicolas SD, Leflon M, Monod H, Eber F, Coriton O, Huteau V, Chevre AM, Jenczewski E: Genetic regulation of meiotic cross-overs between related genomes in Brassica napus haploids and hybrids. Plant Cell 2009, 21(2):373-385

21. Cifuentes M, Eber F, Lucas MO, Lode M, Chevre AM, Jenczewski E: Repeated polyploidy drove different levels of crossover suppression between homoeologous chromosomes in Brassica napus allohaploids. Plant Cell 2010, 22(7):2265-2276.

22. Panjabi P, Jagannath A, Bisht NC, Lakshmi KL, Sharma S, Gupta V, Pradhan AK, Pental D: Comparative mapping of Brassica juncea and Arabidopsis thaliana using Intron Polymorphism (IP) markers: Homoeologous relationships, diversification and evolution of the A, B and C Brassica genomes. BMC Genomics 2008, 9:113.

23. Beilstein MA, Al-Shehbaz IA, Kellogg EA: Brassicaceae phylogeny and trichome evolution. Am J Bot 2006, 93(4):607-619.

24. Yang YW, Lai KN, Tai PY, Li WH: Rates of nucleotide substitution in angiosperm mitochondrial DNA sequences and dates of divergence between Brassica and other angiosperm lineages. J Mol Evol 1999, 48(5):597-604

25. Lysak MA, Koch MA, Pecinka A, Schubert I: Chromosome triplication found across the tribe Brassiceae. Genome Res 2005, 15(4):516-525.

26. Inaba R, Nishio T: Phylogenetic analysis of Brassiceae based on the nucleotide sequences of the S-locus related gene, SLR1. Theor Appl Genet 2002, 105(8):1159-1165.

27. Parkin IA, Gulden SM, Sharpe AG, Lukens L, Trick M, Osborn TC, Lydiate DJ: Segmental structure of the Brassica napus genome based on comparative analysis with Arabidopsis thaliana. Genetics 2005, 171(2):765-781.

28. Schranz ME, Lysak MA, Mitchell-Olds T: The ABC's of comparative genomics in the Brassicaceae: building blocks of crucifer genomes. Trends Plant Sci 2006, 11(11):535-542.

29. O'Neill CM, Bancroft I: Comparative physical mapping of segments of the genome of Brassica oleracea var. alboglabra that are homoeologous to sequenced regions of chromosomes 4 and 5 of Arabidopsis thaliana. Plant J 2000, 23(2):233-243.

30. Park JY, Koo DH, Hong CP, Lee SJ, Jeon JW, Lee SH, Yun PY, Park BS, Kim HR, Bang JW, et al: Physical mapping and microsynteny of Brassica rapa ssp. pekinensis genome corresponding to a $222 \mathrm{kbp}$ gene-rich region of Arabidopsis chromosome 4 and partially duplicated on chromosome 5. Mol Genet Genomics 2005, 274(6):579-588

31. Rana D, van den Boogaart T, O'Neill CM, Hynes L, Bent E, Macpherson L, Park JY, Lim YP, Bancroft I: Conservation of the microstructure of genome segments in Brassica napus and its diploid relatives. Plant J 2004, 40(5):725-733.

32. Town CD, Cheung F, Maiti R, Crabtree J, Haas BJ, Wortman JR, Hine EE, Althoff R, Arbogast TS, Tallon LJ, et al: Comparative genomics of Brassica olerace $a$ and Arabidopsis thaliana reveal gene loss, fragmentation, and dispersal after polyploidy. Plant Cell 2006, 18(6):1348-1359.

33. Yang TJ, Kim JS, Kwon SJ, Lim KB, Choi BS, Kim JA, Jin M, Park JY, Lim MH, Kim HI, et al: Sequence-level analysis of the diploidization process in the triplicated FLOWERING LOCUS C region of Brassica rapa. Plant Cell 2006, 18(6):1339-1347.

34. Cheung F, Trick M, Drou N, Lim YP, Park JY, Kwon SJ, Kim JA, Scott R, Chris Pires J, Paterson AH, et al: Comparative analysis between homoeologous genome segments of Brassica napus and its progenitor species reveals extensive sequence-level divergence. Plant Cell 2009, 21(7):1912-1928.

35. Bohuon EJR, Keith DJ, Parkin IAP, Sharpe AG, Lydiate DJ: Alignment of the conserved C genomes of Brassica oleracea and Brassica napus. Theor Appl Genet 1996, 93(5):833-839.
36. Choi SR, Teakle GR, Plaha P, Kim JH, Allender CJ, Beynon E, Piao ZY, Soengas $P$, Han $T H$, King GJ, et al: The reference genetic linkage map for the multinational Brassica rapa genome sequencing project. Theor Appl Genet 2007, 115(6):777-792.

37. Delourme R, Falentin C, Huteau V, Clouet V, Horvais R, Gandon B, Specel S, Hanneton L, Dheu JE, Deschamps M, et al: Genetic control of oil content in oilseed rape (Brassica napus L.). Theor Appl Genet 2006, 113(7):1331-1345.

38. Mun JH, Kwon SJ, Yang TJ, Kim HS, Choi BS, Baek S, Kim JS, Jin M, Kim JA, Lim $\mathrm{MH}$, et al: The first generation of a BAC-based physical map of Brassica rapa. BMC Genomics 2008, 9:280.

39. Qiu D, Morgan C, Shi J, Long Y, Liu J, Li R, Zhuang X, Wang Y, Tan X, Dietrich $E$, et al: A comparative linkage map of oilseed rape and its use for QTL analysis of seed oil and erucic acid content. Theor App/ Genet 2006, 114(1):67-80.

40. Johnston JS, Pepper AE, Hall AE, Chen ZJ, Hodnett G, Drabek J, Lopez R, Price HJ: Evolution of genome size in Brassicaceae. Ann Bot 2005, 95(1):229-235

41. Luo MC, Thomas C, You FM, Hsiao J, Ouyang S, Buell CR, Malandro M McGuire PE, Anderson OD, Dvorak J: High-throughput fingerprinting of bacterial artificial chromosomes using the SNaPshot labeling kit and sizing of restriction fragments by capillary electrophoresis. Genomics 2003, 82(3):378-389.

42. Nelson W, Soderlund C: Integrating sequence with FPC fingerprint maps. Nucleic Acids Res 2009, 37(5):e36.

43. Nelson WM, Bharti AK, Butler E, Wei F, Fuks G, Kim H, Wing RA, Messing J, Soderlund $C$ : Whole-genome validation of high-information-content fingerprinting. Plant Physiol 2005, 139(1):27-38.

44. Bancroft I: Insights into the structural and functional evolution of plant genomes afforded by the nucleotide sequences of chromosomes 2 and 4 of Arabidopsis thaliana. Yeast 2000, 17(1):1-5.

45. Wang $X$, Wang H, Wang J, Sun R, Wu J, Liu S, Bai Y, Mun JH, Bancroft I, Cheng F, et al: The genome of the mesopolyploid crop species Brassica rapa. Nat Genet 2011, 43(10):1035-1039.

46. Trick M, Kwon SJ, Choi SR, Fraser F, Soumpourou E, Drou N, Wang Z, Lee SY, Yang TJ, Mun JH, et al: Complexity of genome evolution by segmental rearrangement in Brassica rapa revealed by sequence-level analysis. BMC Genomics 2009, 10:539.

47. Kurtz S, Phillippy A, Delcher AL, Smoot M, Shumway M, Antonescu C, Salzberg SL: Versatile and open software for comparing large genomes. Genome Biol 2004, 5(2):R12.

48. Freeling M, Woodhouse MR, Subramaniam S, Turco G, Lisch D, Schnable JC: Fractionation mutagenesis and similar consequences of mechanisms removing dispensable or less-expressed DNA in plants. Curr Opin Plant Biol 2012, 15(2):131-139.

49. Freeling M: Bias in plant gene content following different sorts of duplication: tandem, whole-genome, segmental, or by transposition. Annu Rev Plant Biol 2009, 60:433-453.

50. Hemingway JS: Mustards. In Evolution of Crop Plants. Edited by Simmonds NW. London and New York: Longman; 1976:56-59.

51. Chauhan JS, Singh KH, Singh W, Satyanshu K: Hundred years of rapeseedmustard breeding in India: accomplishments and future strategies. Indian J of Agr Sci 2011, 81(12):1093-1109.

52. Plieske J, Struss D, Robbelen G: Inheritance of resistance derived from the Bgenome of Brassica against Phoma lingam in rapeseed and the development of molecular markers. Theor App/ Genet 1998, 97(5-6):929-936.

53. Saal B, Brun H, Glais I, Struss D: Identification of a Brassica juncea-derived recessive gene conferring resistance to Leptosphaeria maculans in oilseed rape. Plant Breeding 2004, 123(6):505-511.

54. Saal B, Struss D: RGA- and RAPD-derived SCAR markers for a Brassica Bgenome introgression conferring resistance to blackleg in oilseed rape. Theor Appl Genet 2005, 111(2):281-290.

55. Warwick SI, Sauder CA, Mayer MS, Al-Shehbaz IA: Phylogenetic relationships in the tribes schizopetaleae and thelypodieae (brassicaceae) based on nuclear ribosomal ITS region and plastid ndhF DNA sequences. Botany 2009, 87(10):961-985.

56. Uyenoyama MK: A generalized least-squares estimate for the origin of sporophytic self-incompatibility. Genetics 1995, 139(2):975-992.

57. Mandakova T, Lysak MA: Chromosomal phylogeny and karyotype evolution in $\mathrm{x}=7$ crucifer species (Brassicaceae). Plant Cell 2008, 20(10):2559-2570 
58. Chevre AM, Brun H, Eber F, Letanneur JC, Vallee P, Ermel M, Glais I, Li H, Sivasithamparam K, Barbetti MJ: Stabilization of resistance to Leptosphaeria maculans in Brassica napus - B. juncea recombinant lines and its introgression into spring-type Brassica napus. Plant Disease 2008, 92(8):1208-1214

59. Axelsson T, Bowman CM, Sharpe AG, Lydiate DJ, Lagercrantz U: Amphidiploid Brassica juncea contains conserved progenitor genomes. Genome 2000, 43(4):679-688.

60. Griffiths S, Sharp R, Foote TN, Bertin I, Wanous M, Reader S, Colas I, Moore G: Molecular characterization of $\mathrm{Ph} 1$ as a major chromosome pairing locus in polyploid wheat. Nature 2006, 439(7077):749-752.

61. Garcia-Mas J, Benjak A, Sanseverino W, Bourgeois M, Mir G, Gonzalez VM, Henaff E, Camara F, Cozzuto L, Lowy E, et al: The genome of melon (Cucumis melo L.). P Natl Acad Sci USA 2012, 109(29):11872-11877.

62. Tang H, Woodhouse MR, Cheng F, Schnable JC, Pedersen BS, Conant G, Wang X, Freeling M, Pires JC: Altered patterns of fractionation and exon deletions in Brassica rapa support a two-step model of paleohexaploidy. Genetics 2012, 190(4):1563-1574.

63. Sambrook J, Russell DW: Molecular Cloning, A Laboratory Manual. 3rd edition. Woodbury, NY: Cold Spring Harbor Laboratory Press; 2001.

64. Church GM, Gilbert W: Genomic sequencing. P Natl Acad Sci USA 1984, 81(7):1991-1995

65. You FM, Luo MC, Gu YQ, Lazo GR, Deal K, Dvorak J, Anderson OD: GenoProfiler: batch processing of high-throughput capillary fingerprinting data. Bioinformatics (Oxford, England) 2007, 23(2):240-242.

66. Majoros WH, Pertea M, Salzberg SL: TigrScan and GlimmerHMM: two open source ab initio eukaryotic gene-finders. Bioinformatics 2004, 20(16):2878-2879.

67. Thompson JD, Higgins DG, Gibson TJ: CLUSTAL W: improving the sensitivity of progressive multiple sequence alignment through sequence weighting, position-specific gap penalties and weight matrix choice. Nucleic Acids Res 1994, 22(22):4673-4680

68. Yang Z: PAML 4: phylogenetic analysis by maximum likelihood. Mol Biol Evol 2007, 24(8):1586-1591.

69. Koch MA, Haubold B, Mitchell-Olds T: Comparative evolutionary analysis of chalcone synthase and alcohol dehydrogenase loci in Arabidopsis, Arabis, and related genera (Brassicaceae). Mol Biol Evol 2000, 17(10):1483-1498.

70. Capella-Gutierrez S, Silla-Martinez JM, Gabaldon T: trimAl: a tool for automated alignment trimming in large-scale phylogenetic analyses. Bioinformatics 2009, 25(15):1972-1973.

71. Smith SA, Dunn CW: Phyutility: a phyloinformatics tool for trees, alignments and molecular data. Bioinformatics (Oxford, England) 2008 24(5):715-716

72. Price MN, Dehal PS, Arkin AP: FastTree 2-approximately maximumlikelihood trees for large alignments. PLoS One 2010, 5(3):e9490.

73. Huson DH, Scornavacca C: Dendroscope 3: an interactive tool for rooted phylogenetic trees and networks. Syst Biol 2012, 61(6):1061-1067.

doi:10.1186/1471-2164-14-250

Cite this article as: Navabi et al: Conserved microstructure of the Brassica B Genome of Brassica nigra in relation to homologous regions of Arabidopsis thaliana, B. rapa and B. oleracea. BMC Genomics 2013 14:250.

\section{Submit your next manuscript to BioMed Central and take full advantage of:}

- Convenient online submission

- Thorough peer review

- No space constraints or color figure charges

- Immediate publication on acceptance

- Inclusion in PubMed, CAS, Scopus and Google Scholar

- Research which is freely available for redistribution 